LAWRENCE LIVERMORE NATIONAL LABORATORY

\title{
Implications of Nanoabsorber Initiators for Damage Probability Curves, Pulselength Scaling and Laser Conditioning
}

M.D. Feit and A.M. Rubenchik

November 1, 2003

SPIE Boulder Damage Symposium XXXV-Annual Symposium on Optical Materials for High Power Lasers Boulder, Colorado

September 22-24, 2003 
This document was prepared as an account of work sponsored by an agency of the United States Government. Neither the United States Government nor the University of California nor any of their employees, makes any warranty, express or implied, or assumes any legal liability or responsibility for the accuracy, completeness, or usefulness of any information, apparatus, product, or process disclosed, or represents that its use would not infringe privately owned rights. Reference herein to any specific commercial product, process, or service by trade name, trademark, manufacturer, or otherwise, does not necessarily constitute or imply its endorsement, recommendation, or favoring by the United States Government or the University of California. The views and opinions of authors expressed herein do not necessarily state or reflect those of the United States Government or the University of California, and shall not be used for advertising or product endorsement purposes. 


\title{
Implications of nanoabsorber initiators for damage probability curves, pulselength scaling and laser conditioning
}

\author{
M.D. Feit and A.M. Rubenchik \\ University of California \\ Lawrence Livermore National Laboratory \\ P.O. Box 808 L-491 \\ Livermore, CA 94550
}

\begin{abstract}
Laser damage of large optics initiates at material imperfections. Absorbers of very small, nanoscale size are possible initiators. We will analyze experimental implications of assuming that the damage is initiated by a size distribution of nanoabsorbers. We will demonstrate that the model predicts damage fluence pulselength scaling consistent with experiment. The size distribution of nanoabsorbers is related to the resulting damage site density and to the shape of the damage probability curve (S-curve). Conditioning of KDP crystals can be explained within the same model. The relative efficiency of various conditioning strategies is discussed
\end{abstract}

\section{Introduction}

The damage of optical elements is one of the main limiting factors for any large laser system. This is especially true for UV optics due to enhanced absorption at high frequency. The understanding of damage mechanisms can impact system design, choice of operational parameters and technology development.

The idea that damage is initiated by small absorbers was suggested many years ago ${ }^{1,2,3,4}$. However, there is no general discussion of the overall results of such a model. We present a first attempt at such a discussion in the present paper.

We start with a summary of the main facts about damage in high quality optical materials:

1. The observed damage thresholds in fused silica and KDP are 30-50 times lower than estimated intrinsic thresholds ${ }^{5,6}$. Note that intrinsic damage, due to mechanisms such as pure multi-photon absorption and dielectric breakdown, is determined by light intensity only, not fluence. These lower bounds for the intrinsic damage intensity are $80 \mathrm{Gw} / \mathrm{cm}^{2}$ for $1 \mu \mathrm{m}$ light and $150 \mathrm{Gw} / \mathrm{cm}^{2}$ for $350 \mathrm{~nm}$ radiation, respectively.

2. Damage is localized and probabilistic; damage spots are distributed more or less uniformly. The probability to find at least one damage site in volume $\mathrm{V}$ is related to the damage density $\mathrm{c}(\mathrm{F})$ by ${ }^{7,8,4}$

$$
P(F)=1-e^{-c(F) V}
$$

When the observational volume is much smaller than $1 / \mathrm{c}$, it can be free of damaging defects and the resulting damage will be intrinsic. These predictions are consistent with recent measurements ${ }^{9}$ in which the damage threshold produced by a tightly focused beam was studied. The focal volume was of size of a few microns. In these experiments, the threshold was found to depend only on laser intensity for pulse durations from $40 \mathrm{psec}$ to $30 \mathrm{nsec}$. Additionally, the threshold intensity found was higher than the theoretical lower bounds estimated in refs. $[5,6]$. 
3. The damage threshold fluence scales with pulse duration $\tau$ as $\tau^{\mathrm{m}}$, with $\mathrm{m} \leq 0.5$. The specific exponent can be different for specific experiments and materials ${ }^{10}$.

4. Damage precursors have generally not been found by visual inspection and therefore are presumably small.

5. Damage initiation is accompanied by plasma formation and high temperatures. ${ }^{11}$

6. Bulk KDP damage size increases and the pinpoint density decreases with pulse duration. ${ }^{12,13}$

We will show below that all of these observations are consistent with the assumption that damage is initiated by small absorbers.

In the first part of this paper, we discuss absorption by small particles, particularly how absorption varies with particle size for different materials. Then, we calculate the heating of such particles by laser radiation. When the temperature of the inclusion reaches some critical value $T_{c}$, a thermal explosion takes place. The material surrounding the inclusion becomes absorbing and is transformed into a growing plasma fireball. The release of the energy stored in the fireball produces macroscopic damage, i.e. cracking and other irreversible modifications of the material.

Assuming that the material contains absorbers with a size distribution, we find that for any given pulse duration, a size most susceptible to damage exists. The dependence of this size on pulse duration determines the pulselength scaling of the damage threshold. The observable damage distribution is related to the underlying distribution of damage initiators.

It is well known that KDP crystals can be conditioned ${ }^{12,13}$. Prior irradiation at less than operational levels increases the resistance of KDP to bulk damage. We will discuss a possible conditioning mechanism within the nanoabsorber model and examine aspects of an optimal conditioning strategy following from this model.

\section{Small Particle Absorption}

Damage of large fused silica optics typically takes place on the surface. Evidently, the damage is associated with surface finishing. For example, particles of polishing material can be trapped in microcracks in the subsurface mechanically damaged layer created by polishing. Some common materials like $\mathrm{CeO}_{2}$ have strong UV absorption and are dangerous from this viewpoint. Formation of cracks during polishing process is associated with the formation of free surfaces. During this process, oxygen can escape forming oxygen deficient clusters, which can absorb UV light. This effect can be especially pronounced for small microparticles chipped out during crack propagation since they have a high surface to volume ratio.

The power $\mathrm{Q}$ absorbed by a particle is $\mathrm{Q} \square \alpha \mathrm{I} \pi \mathrm{a}$. The absorptivity $\alpha=\sigma / \pi \mathrm{a}^{2}$, where $\sigma$ is the absorption cross section and a the particle radius, is given ${ }^{14}$ for a small particle with radius very much smaller than the laser wavelength by

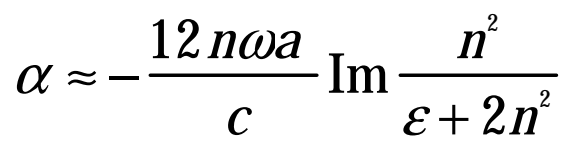

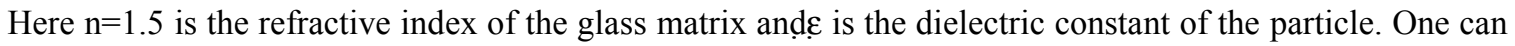
see that absorptivity initially increases linearly with particle radius. This expression is not applicable when the particle size becomes comparable with the wavelength of light, n $\omega \mathrm{a} / \mathrm{c} \sim 1$. In this case, absorptivity 
may be calculated using Mie theory. In Figs. 1 and 2, we show absorptivity as a function of particle size for several materials.

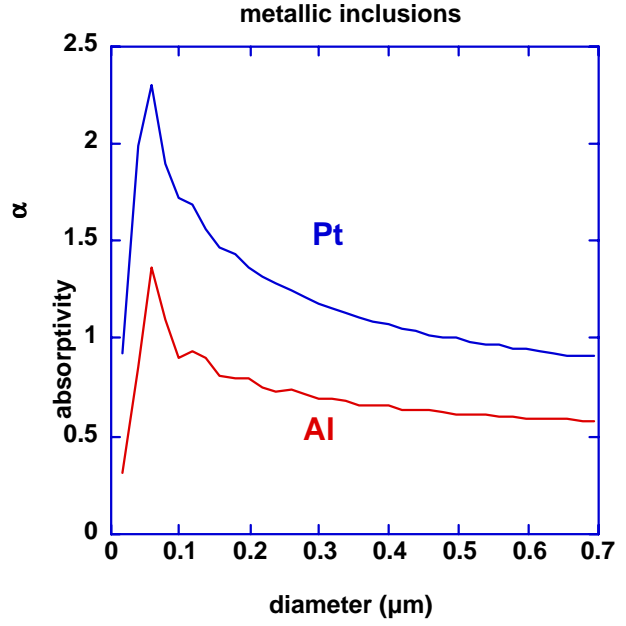

Fig.1: Calculated absorptivity at $355 \mathrm{~nm}$ vs inclusion diameter for metallic absorbers.

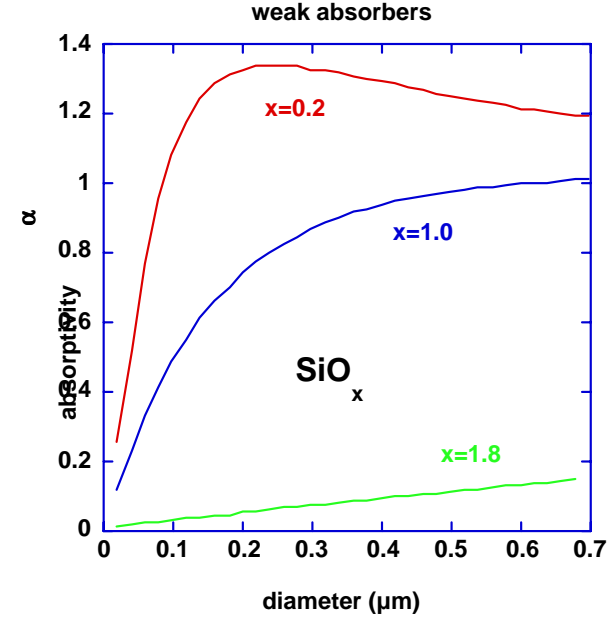

Fig.2: Calculated $\mathrm{SiO}_{\mathrm{x}}$ absorptivity vs. diameter for several amounts of oxygen deficiency.

In Fig.1, we present the absorptivity of strong absorbers, viz. Al and Pt inclusions. One can see that for metals, $\alpha$ can be treated as approximately constant for inclusions with diameter greater than $100 \mathrm{~nm}$. In Fig.2, the absorptivity for $\mathrm{SiO}_{\mathrm{x}}$ clusters is shown. For strongly absorbing clusters (x small), with high oxygen deficiency, the constant absorptivity model is adequate. For weaker absorbers, the absorptivity increases with inclusion size up to micron-sized particles. At this point, we do not know the nature of the absorbers initiating damage, but analysis of experiments, as we will show later, allows the elimination of some candidates.

\section{Thermal Model of Absorption}

We assume that damage is initiated by laser light absorption in nanoparticles. When the particle surface temperature reaches some critical value, a thermal explosion produces a plasma fireball, which then leads to damage of the material. To determine the laser fluence needed to heat the particle up to the critical temperature we must solve the appropriate heat equation. Assuming the thermal conductivity of the absorbing particle is high compared to that of the surrounding substrate, we solve

$$
\frac{\partial T}{\partial t}=D_{0} \Delta T
$$

The boundary condition at $\mathrm{r}=\mathrm{a}$ is

$$
\alpha l(t)=-4 \kappa \frac{\partial T}{\partial r}+\frac{4}{3} \rho C a \frac{\partial T}{\partial t}
$$

where $\alpha$ is the absorptivity of the particle, which we assume constant, $\mathrm{D}_{0}$ the thermal diffusivity and $\kappa$ the thermal conductivity of the matrix and $\rho$ and $\mathrm{C}$ are the density and heat capacity of the particle. We have assumed that the temperature is homogeneous inside the nanoparticle. This corresponds to the high particle thermal conductivity and is reasonable since the absorber has more free electrons. Also, for weak absorbers the energy deposition is quite homogeneous in the particle, which additionally justifies our assumption. The solution of (2.1) with boundary condition (2.2) gives a simple expression ${ }^{15}$ for the particle temperature $\mathrm{T}$ due to heating by a rectangular laser pulse of duration $\tau$ : 


$$
T=T_{0}\left(1-e^{-\frac{4 D \tau}{a^{2}}}\right) \text { where } T_{0}=\frac{\alpha F a}{4 \kappa \tau} \text { and } D=\frac{3 \kappa}{4 \rho C}
$$

The temperature as a function of absorber size is plotted in Fig.3. One can see that for a fixed pulse duration, there is a particle size most susceptible to heating. Small particles, because of their high surface to volume ratio, are kept cool by heat conduction to the substrate. Large particles contain too much matter to be heated to high temperature during the pulse. The temperature is maximum at $\mathrm{a}^{2} / 4 \mathrm{D}=0.81 \tau$ (See Fig.3). This corresponds to the most dangerous absorber size of $2 a=3.6 \sqrt{ } \mathrm{D} \tau(\mathrm{D}=3 \kappa / 4 \rho C)$. The effective thermal diffusivity $\mathrm{D}$ in this relation is not very sensitive to the exact material. For example, with a $3 \mathrm{nsec}$ rectangular pulse, the most susceptible inclusion size is $152 \mathrm{~nm}$ for $\mathrm{SiO}_{\mathrm{x}}, 124 \mathrm{~nm}$ for $\mathrm{Al}$ and $144 \mathrm{~nm}$ for $\mathrm{Zr}$.

The temperature pattern driven by a Gaussian pulse is similar to (2.3). With $\mathrm{I}=\mathrm{I}_{0} \operatorname{Exp}\left[-\pi(\mathrm{t} / \tau)^{2}\right]$, where the definition of pulselength $\tau$ for the Gaussian was chosen such that fluence $\mathrm{F}=\mathrm{I}_{0} \tau$, the maximum temperature is reached at time $\mathrm{t}=0.41 \tau$ for the most susceptible particle size which is still $2 \mathrm{a}=3.6 \sqrt{\mathrm{D} \tau} \tau$. Note that the pulselength scaling of size, $a \sim \sqrt{ } \mathrm{D} \tau$, is independent of the pulse shape.

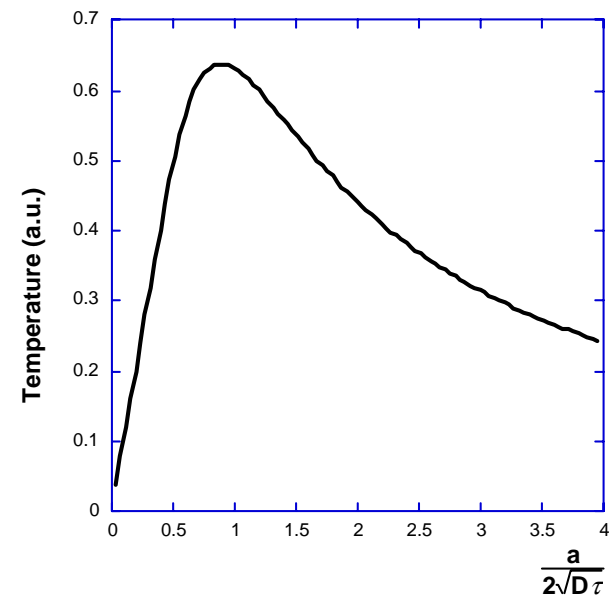

Fig.3: Inclusion temperature induced by a rectangular laser pulse for absorbers of different sizes. Particle size is normalized by the thermal length $\sqrt{D} \tau$.

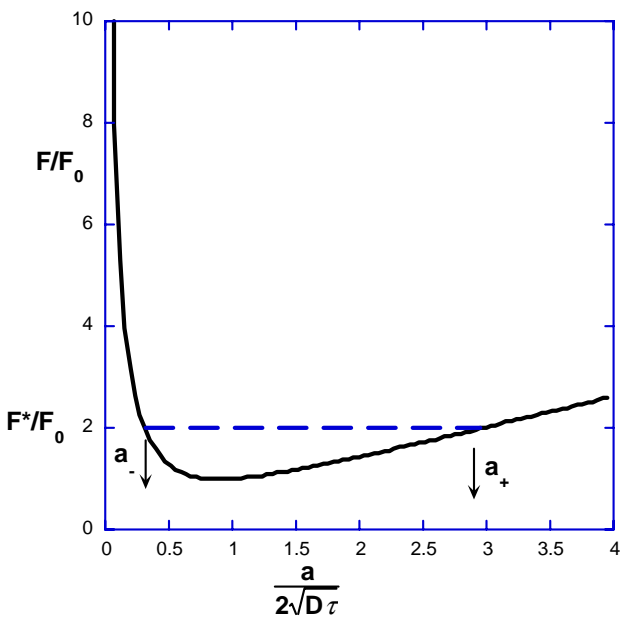

Fig.4: Normalized fluence damage threshold F/F0 vs. normalized particle size. At fluence $\mathrm{F}^{*}$, particles with sizes $\mathrm{a}_{-}<\mathrm{a}<\mathrm{a}_{+}$are above their threshold. The resulting damage density is found by integrating the precursor density $n(a)$ between sizes $a_{-}$and $a_{+}$.

The temperature reached by the most susceptible absorbers heated by a rectangular pulse is given by

$$
T=\frac{0.16 \alpha F \sqrt{D}}{\kappa \sqrt{\tau}}
$$

The maximum temperature is the same for the Gaussian pulse defined above.

\section{Scaling for damage thresholds and defect density}


We assume that damage takes place when the absorber temperature reaches the critical value $T_{c}$. The electron energy bandgap in fused silica collapses at $\mathrm{T} \sim 2500{ }^{\circ} \mathrm{K}$, but due to the nonlinearity of thermal conduction, radiation transport and absorption, $\mathrm{T}_{\mathrm{c}}$ might be even lower than this estimate.

For threshold fluence $\mathrm{F}_{0}$ to produce this temperature, we find from (2.4)

$$
F_{0}=\frac{6.3 \mathrm{~T}_{\mathrm{c}} \kappa \sqrt{\tau}}{\alpha \sqrt{D}}
$$

No damage occurs at fluences below $\mathrm{F}_{0}$. The damage density increases rapidly at fluences above $\mathrm{F}_{0}$ so it is natural to treat $\mathrm{F}_{0}$ as the threshold fluence. One sees from (3.1) that $\mathrm{F}_{0} \sim \sqrt{\tau}$, consistent with experiments. We comment on the origin of deviations from the square root of pulselength below.

For pulse duration of $3 \mathrm{nsec}$ and particle radius $70 \mathrm{~nm}$, Eq.(3.1) predicts

$$
F_{0}=\frac{0.18}{\alpha} \mathrm{J} / \mathrm{cm}^{2}
$$

For a typical damage fluence $\mathrm{F}_{0}=6 \mathrm{~J} / \mathrm{cm}^{2}$ the above expressions imply that the absorptivity is small, $\alpha \sim 0.03$. This leads us to conclude that the inclusions responsible for damage initiation cannot be strong absorbers like metals.

In the above, we assumed constant absorptivity $\alpha$. However, note that the result (2.3) remains valid when absorptivity depends on inclusion radius a. From the above, absorptivity should be a weak increasing function of particle size. The simplest case is power law variation. Assuming that $\alpha \sim a^{p}$, where $p<1$, one sees that the size of the most susceptible inclusions still varies as $\sqrt{D} \tau$, only the numerical coefficient changes. As an example, for $\mathrm{p}=0.4$ the size of most susceptible particles is $30 \%$ larger than it is for $\mathrm{p}=0$. The size dependence of absorptivity results in a slower pulse duration scaling of threshold fluence $\mathrm{F}_{0}$, viz. $\mathrm{F}_{0} \sim \tau^{(1-\mathrm{p}) / 2}$. For example, $\mathrm{p}=0.4$ corresponds to $\mathrm{F}_{0} \sim \tau^{0.3}$.

At fluences above $\mathrm{F}_{0}, \mathrm{~F}>\mathrm{F}_{0}$, inclusions with a range of sizes $\mathrm{a}_{-}<\mathrm{a}<\mathrm{a}_{+}$will be above their thresholds. (see Fig.4). The underlying size distribution $\mathrm{n}(\mathrm{a})$ is related to the damage density $\mathrm{c}(\mathrm{F})$ derivable from experiment (see Eq. I.1) by

$$
c(F)=\int_{a_{-}}^{a_{t}} n(a) d a
$$

Experiments indicate a strong dependence of $\mathrm{c}(\mathrm{F})$ on fluence $\mathrm{F}$, typically a power law or exponential. This suggests that $\mathrm{n}$ (a) may also be strongly dependent on a, perhaps having a form like

$$
n(a)=\frac{N}{a^{m}}
$$

In any case, the important point is that there are more small absorbers than large absorbers. If this is the case, when fluence is above $\mathrm{F}_{0}$, the contribution to $\mathrm{c}(\mathrm{F})$ in $\mathrm{Eq}$.(3.2) from small absorbers will dominate. The interpretation is that as fluence is increased the additional damage initiation is due to the more numerous smaller precursors. Because $\mathrm{a}_{\text {_ }}$ scales as

$$
a_{-}=a_{0} \quad g\left(\frac{F}{F_{0}}\right)
$$


and $\mathrm{a} 0=1.8 \sqrt{\mathrm{D}} \tau$, it follows that $\mathrm{c}(\mathrm{F})$ will be a strong function of fluence and that the damage density will decrease at longer pulselengths. ${ }^{12}$

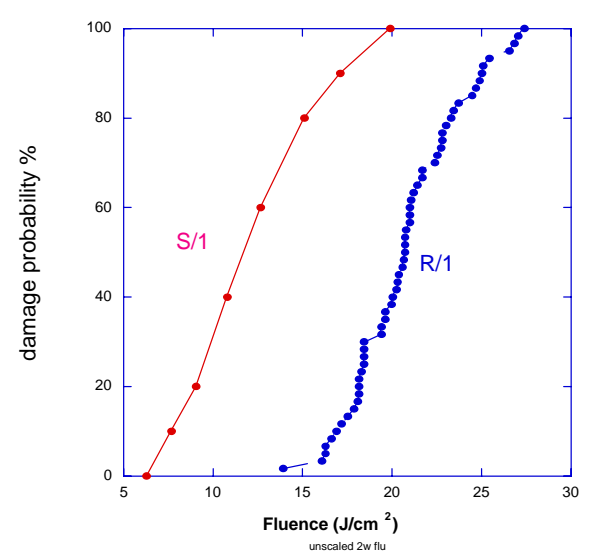

Fig.5: Comparison of typical $\mathrm{S} / 1$ and $\mathrm{R} / 1$ laser damage tests on frequency conversion material. The curve shifts to higher fluence in the $\mathrm{R} / 1$ test because of conditioning.

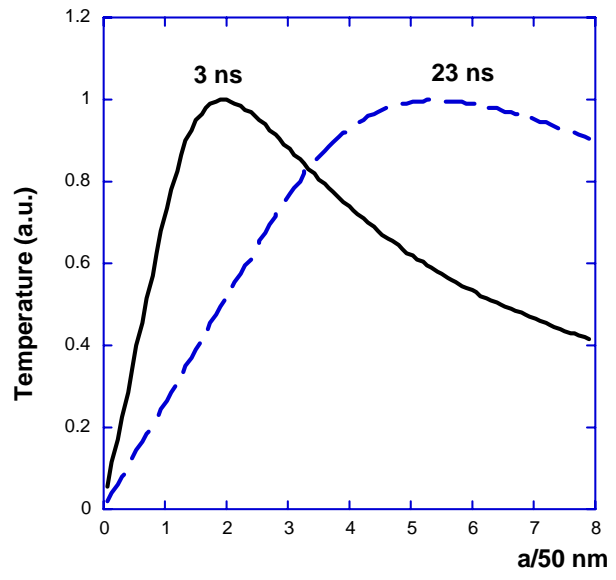

Fig.6: The effect of pulse duration on the size dependence of the relative temperature reached by laser pulse heating.

\section{KDP Conditioning}

It is well known that prior irradiation of KDP crystals by the pulses of lower than operational fluence improves the damage strength of the crystals. The effects of conditioning can be seen in the comparison of $\mathrm{S} / 1$ and $\mathrm{R} / 1$ damage tests(Fig.5). One sees that damage susceptibility in the $\mathrm{R} / 1$ test, where the fluence gradually ramps up to the damaging fluence, better than that in the $\mathrm{S} / 1$ test in which a single fluence is used.

The process of conditioning can be potentially understood within the small absorber model as follows. At low fluence, inclusions are heated up to temperatures sufficient to melt the surrounding substrate material and disperse the inclusion. The difference in the densities of melted and solid KDP produces high stresses that lead to mixing of the inclusion material with the native material. Because the melting temperature in KDP is far below the bandgap collapse temperature, the heated substrate material is still transparent and well below the threshold for thermal explosion. It is possible to show ${ }^{16}$ that when the melt radius exceeds some critical value, a "pan cake" shape of the melt becomes energetically favored over a spherical shape since it decreases elastic energy. When the melted volume becomes large enough, a jet forms. The resulting melt motion will enhance the decomposition of the inclusion. When the absorbing material is distributed over a volume a few times the thermal length, the inclusions becomes benign. That is, conditioning takes place. It is possible to have inclusions that remain nearly solid up to bandgap collapse temperatures. In this case, the conditioning mechanism outlined here will not be effective for them. The damage evidenced in $\mathrm{R} / 1$ tests may be caused by such inclusions

It is convenient to condition crystals for large laser systems offline. The ideal situation is to condition with pulses of equal pulselength to those expected to be used in operations. However, large systems may generate laser pulses over a range of pulselengths. The question then arises as to what pulselength to use for conditioning if only one conditioning pulselength is used. 
As noted above, conditioning, within the small absorber model, means heating the absorber and surrounding material up to a temperature $\mathrm{T}_{\mathrm{m}}<\mathrm{T}_{\mathrm{c}}$ at which mixing can occur without thermal explosion (i.e. damage). Inclusions best conditioned with long pulses are not those most susceptible for short pulses. In Fig.6, we plot the relative temperature as a function of particle size reached by absorbers heated by pulses of durations 3 and $23 \mathrm{nsec}$. The issue is we have to avoid damage at the inclusions most susceptible at the given pulselength (i.e. have fluence low enough) while still achieving adequate conditioning for other inclusions that are most susceptible at another pulselength (i.e. have fluence high enough). Because the curves shown are steeper on the small size end, corresponding to shorter pulses, it is advantageous to condition with shorter pulses. To make this point more quantitatively, consider two pulses with durations $t_{1}>t_{2}$ and consider an inclusion with size a most damage susceptible for pulse duration $t_{2}$, i.e. $a=1.8 \sqrt{ } \mathrm{Dt}_{2}$. With fluence $\mathrm{F}_{2}$ and pulse duration the inclusion will heat up to temperature $\mathrm{T}$

$$
T=\frac{0.16 \alpha F_{2} \sqrt{D}}{\kappa \sqrt{t_{2}}}
$$

To heat the same inclusion to the same temperature using a pulse with duration $t_{1}$ requires the fluence $F$

$$
F=\frac{0.63 t_{1}}{t_{2}\left(1-e^{-\frac{1.2 t_{2}}{t_{2}}}\right)} F_{2} \approx \frac{0.63 t_{1}}{t_{2}} F_{2}
$$

For pulse durations $\mathrm{t}_{1}=23 \mathrm{nsec}$ and $\mathrm{t} 2=3 \mathrm{nsec}, \mathrm{F}=4.8 \mathrm{~F}_{2}$, which is 1.7 times larger then $\mathrm{F}=2.8 \mathrm{~F}_{2}$ which might be expected from conventional $\mathrm{F} \sim V_{\mathrm{t}}$ scaling. This fluence might be high enough to initiate surface damage thus decreasing the efficiency of the bulk damage conditioning process. For a Gaussian pulse, the calculations gives a very similar result, $\mathrm{F}=4.7 \mathrm{~F}_{2}$.

\section{CONCLUSION}

We demonstrated that the model of small-absorber initiated damage is consistent with experimental data on damage and conditioning. The model predicts pulselength scaling of damage threshold, can account for KDP conditioning by absorber mixing with surrounding material, and is consistent with the locality, smallness, plasma generation and statistical nature of damage initiation.

The nature of the initiators, e.g. in KDP, is still unknown, although comparison of the model with experimental data implies some restrictions on the possible candidates. Damage behavior at different frequencies, and with different pulse durations can provide further insights into both the nature of the initiators and their distribution function.

Such a model can help optimize conditioning protocols for frequency conversion crystals.

\section{Acknowledgement}

This work was performed under the auspices of the U.S. Department of Energy by University of California Lawrence Livermore National Laboratory under contract No. W-7405-Eng-48

\section{References}

\footnotetext{
${ }^{1}$ 1. R.W.Hopper, D.P.Uhlmann. "Mechanism of inclusion damage in laser glass.," Appl.Phys.41,40234037 (1970)
} 
${ }^{2}$ M.R.Lange, J.K.McIver, A.H.Guenther, T.W.Walker, "Pulsed laser induced damage of an optical material with a spherical inclusion", Damage in Laser Materials 1982, Nat. Bur. Stand. Spec. Pub. 669, 380-6, (1984)

${ }^{3}$ M.D. Feit, A.M. Rubenchik, M. Runkel, "Analysis of bulk DKDP damage distribution, obscuration and pulse length dependence", Proceedings XXXII Annual Symposium on Optical Materials for High-Power Lasers, Boulder, Co, Oct.16-18, 2000,SPIE-4347, 383-388 (2001)

${ }^{4}$ S.I. Anisimov, V.A. Khokhlov "Instabilities in Laser-matter interaction", CRC Press Boca Raton, (1999)

${ }^{5}$ B. C. Stuart, M. D. Feit, S. Herman, A. M. Rubenchik, B. W. Shore, and M. D. Perry, "Nanosecond to femtosecond laser-induced breakdown in dielectrics", Phys. Rev. B 53, 1749 (1996)

${ }^{6}$ A.M. Rubenchik, M.D. Feit, "Initiation, growth and mitigation of UV laser induced damage in fused silica", Proceedings XXXII Annual Symposium on Optical Materials for High-Power Lasers, Boulder, Co, Oct.16-18, 2000, SPIE-4347, 79-95 (2001)

${ }^{7}$ V.V. Azarov, L.V. Atroschchenko, Yu. K. Danileiko, M.I. Kolybaeva, Yu. P. Minaev, V.N. Nikolaev, A.V. Sidorin and B.I. Zakharkin, "Influence of structure defects on the internal optical strength of KDP single crystals", Sov. J. Quant. Electr. 15, 89-90 (1985)

${ }^{8}$ M.D. Feit, A.M. Rubenchik, M.R. Kozlowski, F. Genin, L. Sheehan, S. Schwartz "Extrapolation of damage test data to predict performance of large-area NIF optics at $355 \mathrm{~nm}$ ", Proceedings XXX Annual Symposium on Optical Materials for High-Power Lasers, SPIE-3578, . 226-231, (1999)

${ }_{9}^{9}$ O. Efimov, "Intrinsic and multiple pulse laser-induced damage of transparent dielectrics in the femtonanosecond region", these Proceedings

${ }^{10}$ F. Rainer, F. DeMarco, M. Staggs, M.R. Kozlowski, L.J. Atherton, L.M. Sheehan, ”A historical perspective on fifteen years of laser damage thresholds in LLNL", XXV Annual Symposium on Optical Materials for High-Power Lasers, Proc. SPIE 2114, 9-22,(1993)

${ }^{11}$ C.W. Carr, H.B. Radousky, M. Staggs, A.M. Rubenchik, M.D. Feit, S.G. Demos, “Time-resolved spectroscopic investigation of emission observed during damage in the bulk of fused silica and DKDP crystals", XXXIII Anпиal Symposium on Optical Materials for High-Power Lasers, Boulder, Co, Oct.1-2, 2001, SPIE 4679, 360-365 (2002)

${ }^{12}$ M. Runkel, A.K. Burnham, D. Milam, W. Sell, M.D. Feit and A.M. Rubenchik, "Results of pulse-scaling experiments on rapid-growth DKDP triplers using the Optical Sciences Laser at $351 \mathrm{~nm}$ ", Proceedings XXXII Annual Symposium on Optical Materials for High-Power Lasers, Boulder, Co, Oct.16-18, 2000, SPIE 4347, 359-372 (2001)

${ }^{13}$ M.D. Feit., A.M. Rubenchik, M. Runkel, "Analysis of bulk DKDP damage distribution, obscuration and pulse length dependence", Proceedings XXXII Annual Symposium on Optical Materials for High-Power

Lasers, Boulder, Co, Oct.16-18, 2000, SPIE-4347, 383-388 (2001)

${ }^{14}$ H.C. van der Hulst, Light scattering by small particles, John Wiley and Sons, NY (1957)

${ }^{15}$ C. Chan, "Effective absorption for thermal blooming due to aerosols", Appl.Phys.Lett. 26, 628, (1975)

${ }^{16}$ E. Brener, S. Iordanskii, V. Marchenko, "Elastic effects on the kinetics of a phase transition", Phys.Rev.Lett. 82,.1506 (1999) 